\title{
Characteristics of predominantly right-sided colonic diverticulitis without need for colectomy
}

\author{
Zhi Chen ${ }^{1}$, Bing Zhang ${ }^{1}$, Dan $\mathrm{Wu}^{2^{*}}$ (D) and Ye Jin ${ }^{1}$
}

\begin{abstract}
Background: In China, diverticulitis is more often located in the right colon, mainly in the cecum and ascending colon. Here we study the characteristics of acute colonic diverticulitis and compare various treatments for acute right-sided colonic diverticulitis.
\end{abstract}

Methods: A retrospective analysis of 123 patients with acute colonic diverticulitis treated in our hospital from April 2013 to April 2020, including 114 cases of right-sided colonic diverticulitis, was performed. The characteristics of acute colonic diverticulitis were analyzed, and the therapeutic effects of different treatments for acute right-sided colonic diverticulitis were compared.

Results: 111 cases of caecal and ascending colonic diverticulitis were identified (90.2\% of cases, male to female ratio $2.26: 1$, average age $39.6 \pm 14.4$ years, surgery ratio $24.3 \%$, mean hospital stay $7.4 \pm 4.3$ days, recurrence rate 3.6\%). Three cases of transverse colonic diverticulitis and three cases of descending colonic diverticulitis were found. Six cases of Sigmoid diverticulitis (4.9\% of cases, male to female ratio 1:1, average age $67.7 \pm 4.5$ years, surgery ratio $33.3 \%$, mean hospital stay $11.7 \pm 5.5$ days, recurrence rate $0 \%$ ) were found. 13 patients underwent right-sided colonic diverticulitis resection and repair, while zero patients underwent colectomy. Abdominal drainage was performed in 15 patients with right-sided colonic diverticulitis. There was no significant difference in the length of hospital stay among the three treatments for right-sided colonic diverticulitis $(P=0.05)$. There was no significant difference in the recurrence rate among the three treatments of right-sided colonic diverticulitis $(P=0.358)$. While the recurrence rate of right-sided colonic diverticulitis was only 3.5\%, relapse usually occurred within the first year following treatment.

Conclusions: In our patients, right-sided colonic diverticulitis is more common in young and middle-aged patients than in elderly patients and we see a higher incidence in males. Acute right-sided complex diverticulitis is rare. While non-surgical treatment is preferred for acute right-sided uncomplicated diverticulitis, no significant difference in outcome was observed between the three different treatments we compared. Resection and repair of diverticulum or abdominal drainage can also be used to treat patients with acute uncomplicated diverticulitis.

Keywords: Acute colonic diverticulitis, Right-sided colonic diverticulitis, Operative

\footnotetext{
* Correspondence: 1837343584@qq.com

${ }^{2}$ Department of Anesthesiology, Zhejiang Hospital, No.1229, Gudun Road,

Xihu District, Hangzhou city 310030, Zhejiang province, China

Full list of author information is available at the end of the article
}

(C) The Author(s). 2020 Open Access This article is licensed under a Creative Commons Attribution 4.0 International License, which permits use, sharing, adaptation, distribution and reproduction in any medium or format, as long as you give appropriate credit to the original author(s) and the source, provide a link to the Creative Commons licence, and indicate if changes were made. The images or other third party material in this article are included in the article's Creative Commons licence, unless indicated otherwise in a credit line to the material. If material is not included in the article's Creative Commons licence and your intended use is not permitted by statutory regulation or exceeds the permitted use, you will need to obtain permission directly from the copyright holder. To view a copy of this licence, visit http://creativecommons.org/licenses/by/4.0/ The Creative Commons Public Domain Dedication waiver (http://creativecommons.org/publicdomain/zero/1.0/) applies to the data made available in this article, unless otherwise stated in a credit line to the data. 


\section{Background}

A colonic diverticulum is a benign lesion involving a local protrusion of the colonic wall, and can arise singly or in multiples. It can occur at any position within the colon, but the characteristics of the disease vary greatly in different regions of the world. Colonic diverticulitis is considered to be rare in China. The incidence of diverticulitis is significantly higher in Europe and the United States. In these countries, diverticulitis usually occurs in the left colon, especially in the sigmoid colon. In China, diverticulitis is more often located in the right colon, mainly in the cecum and ascending colon, and the proportion in the sigmoid colon is low. In western, colonic diverticulitis is more often identified in the middle-aged and elderly, but this may not be the case in China $[1,2]$.

\section{Methods}

\section{Patients}

This article retrospectively analyzed data from 123 patients diagnosed with acute colonic diverticulitis treated from April 2013 to April 2020 in Tongde Hospital of Zhejiang Province. The diagnosis was confirmed by abdominal computed tomography (CT), colonoscopy or surgical exploration. 114 cases of right-sided colonic diverticulitis were identified. 13 patients underwent resection and repair of the right-sided colonic diverticulitis and no patients were treated with colectomy. Abdominal drainage was performed in 15 patients with right-sided colonic diverticulitis. 86 patients received completely non-surgical treatment. Cases included 85 male patients and 38 female patients (male to female ratio 2.24: 1, average age $40.0 \pm 15.4$ years). The study was approved by the Ethics Committee of Zhejiang Tongde Hospital.

Patients were excluded using the following exclusion criteria: 1. the patient had not yet recovered or had stopped treatment prematurely before discharge; 2 . the patient's data was incomplete or follow-up information was lost after discharge.

\section{Method of treatment}

Patients were divided into three treatment groups. The surgical treatment group included patients who were treated surgically, by resection and repair of the diverticulum or colectomy. Following surgery this group received antibiotics and fluid replacement as treatment. In the abdominal drainage group, an abdominal drainage tube was placed by laparoscopy. The non-surgical group were treated using dietary changes, antibiotics, rehydration and other non-surgical measures.

Patients were discharged once they 1 . no longer showed signs of abdominal pain or pain was obviously decreased; and 2. were fever-free for the preceding $48 \mathrm{~h}$, and their inflammatory indexes such as white blood cell count (WBC) and C-reactive protein level (CRP) appeared normal.

\section{Data collection}

The patient's age, gender, Hinchey's classification, diverticulum position, leukocyte index and CRP index before admission, treatment method, length of stay and postoperative follow-up of colonic diverticulum were collected.

\section{Statistics}

SPSS17.0 statistical software was used for statistical processing. One-way analysis of variance was used to compare the mean values of multiple groups, chi-square test or fisher test was used for counting data. $P<0.05$ was considered statistically significant.

\section{Results}

111 patients had diverticulitis in the cecum and ascending colon (90.2\%, male to female ratio 2.26:1, mean age $39.6 \pm 14.4$ years, surgery ratio $24.3 \%$, leukocyte increase rate $64.9 \%$, CRP increase rate 85.6\%, 105 cases of Hinchey's grade I and six cases of Hinchey's grade II, average hospital stay $7.4 \pm 4.3$ days, the average follow-up length $33.5 \pm 21.3$ months, recurrence rate $3.6 \%$ ); three cases in the transverse colon were found $(2.4 \%$, male to female ratio $2: 1$, mean age $50.0 \pm 16.7$ years, surgery ratio $33.3 \%$, leukocyte increase rate $66.6 \%$, CRP increase rate $100 \%$, three cases of Hinchey's grade I, average hospital stay $11.3 \pm 4.0$ days, average follow-up length $38.3 \pm 10.2$ months, recurrence rate $33.3 \%$ ); three cases were located in the descending colon $(2.4 \%, 3$ males, mean age $43.3 \pm$ 11.0 years, surgery rate $0 \%$, leukocyte increase rate $100 \%$, CRP increase rate $100 \%$, three cases of Hinchey's grade I, average hospital stay $5.7 \pm 0.6$ days, average follow-up length $44.3 \pm 28.6$ months, recurrence rate $0 \%$ ); six cases in the sigmoid colon were identified (4.9\%, male to female ratio $1: 1$, mean age $67.7 \pm 4.5$ years, surgery ratio $33.3 \%$, leukocyte increase rate $66.7 \%$, CRP increase rate $66.7 \%$, four cases of Hinchey's grade I and two cases of Hinchey's grade II, average hospital stay $11.7 \pm 5.5$ days, average follow-up length $30.5 \pm 19.2$ months, recurrence rate $0 \%)$. The recurrence rate of right-sided colonic diverticulitis was $3.5 \%$, and relapse usually occurred within one year.

Colectomy was not performed for any patients with right-sided colonic diverticulitis. Although six patients were evaluated as Hinchey's grade II after surgery, they were assessed before surgery as Hinchey's grade I. All six can be considered as uncomplicated diverticulitis. There are no significant differences in the three therapeutic treatments effects (Table 1). There were no complications due to intestinal leakage in both the surgical group and the group treated with abdominal drainage. 
Table 1 Comparison of different treatments for acute right-sided colonic diverticulitis

\begin{tabular}{|c|c|c|c|c|c|}
\hline & $\begin{array}{l}\text { Abdominal drainage group } \\
(n=15)\end{array}$ & $\begin{array}{l}\text { Operation group } \\
(n=13)\end{array}$ & $\begin{array}{l}\text { Completely non operative group } \\
(n=86)\end{array}$ & $F / X^{2}$ & $P$ \\
\hline Age(years) & $34.2 \pm 16.2$ & $39.8 \pm 14.6$ & $40.4 \pm 14.1$ & 1.186 & 0.309 \\
\hline Gender & & & & 1.293 & 0.524 \\
\hline male & 9 & 8 & 62 & & \\
\hline female & 6 & 5 & 24 & & \\
\hline $\mathrm{WBC}\left(10^{9} / \mathrm{L}\right)$ & $11.0 \pm 3.8$ & $11.4 \pm 2.6$ & $10.8 \pm 3.8$ & 0.162 & 0.851 \\
\hline CRP (mg/L) & $55.9 \pm 53.8$ & $59.6 \pm 52.2$ & $55.3 \pm 51.3$ & 0.061 & 0.941 \\
\hline Hospital stay (days) & $7.7 \pm 3.1$ & $11.8 \pm 8.6$ & $6.8 \pm 3.0$ & 3.655 & 0.050 \\
\hline Follow-up length (months) & $35.1 \pm 16.7$ & $52.8 \pm 21.1$ & $30.6 \pm 20.4$ & 6.982 & 0.001 \\
\hline Recurrence rate & $6.7 \%$ & $7.6 \%$ & $2.3 \%$ & 1.667 & 0.358 \\
\hline
\end{tabular}

\section{Discussion}

The etiology of colonic diverticulum is currently unknown and this condition generally produces no clinical symptoms. In the United States, $4 \%$ of patients have clinical symptoms, and $15 \%$ have complicated disease [1]. In Europe and the Americas, the incidence of acute left-sided colonic diverticulitis (ALCD) is higher, while acute right-sided colonic diverticulitis (ARCD) is relatively rare, and ALCD is more common in the elderly [2]. Through a retrospective analysis of colonic diverticulitis in our hospital, we found that ARCD is more common in our patients and identified more often in males, while ALCD is rare. The onset age of ARCD in our population is younger when compared to patients in Europe and the United States. This is consistent with other reports from China [3, 4].

Because the most common type of colonic diverticulitis varies greatly in different regions of the world, there are also differences in clinical manifestations and treatment plans. Caecal and ascending colonic diverticulitis predominate in China; especially diverticulitis near the ileocecum which produces clinical symptoms similar to acute appendicitis including metastatic right lower abdominal pain, right lower abdominal fixed tenderness, and disease progression, etc. [3]. Our hospital prefers non-surgical treatment for ARED, and this conservative approach proves worthy. However, $24.6 \%$ of ARCD cases underwent invasive treatment, mainly because ARCD could not be distinguished from acute appendicitis. While all patients underwent CT examination of the abdomen prior to surgery, even experienced doctors can misdiagnose CT images. When we carefully re-analyzed the abdominal CT images after surgery, we found that it was possible to distinguish between ARCD and acute appendicitis. However, ARCD is usually characterized by acute abdominal pain, so it is difficult to rapidly diagnose before surgery. According to the WESE guidelines, ultrasonography is the imaging modality of choice for
ARCD because usually patients are younger and CT imaging poses the risk of exposure to radiation [2]. Cases from our hospital support this author's belief that CT provides advantages over ultrasound when distinguishing acute appendicitis and ARCD. The most common cause of acute abdominal pain in Chinese hospitals is acute appendicitis. Misdiagnosis of an ultrasound may result in emergency surgery. However, we assert that most cases of ARCD do not require surgical treatment, and that abdominal CT can exclude incorrect diagnoses.

Compared with acute appendicitis, we have found that the clinical symptoms of ARCD were milder, and began to resolve more rapidly after treatment. Timely and effective treatment only rarely leads to diffuse peritonitis or intestinal leakage, which inevitably cause surgeons to mistakenly think that ARCD is mild. However, in a number of surgical exploration cases, we found that the ARCD had suppurated and perforated. The affected area was partially wrapped by the greater omentum, so the clinical symptoms were mild. As mentioned earlier, the patient underwent emergency surgery because of a misdiagnosis of acute appendicitis without severe clinical symptoms. Therefore, we suspect that more patients with suppuration and perforation may be found in the non-surgical treatment group. We also found that, preoperatively, it was difficult to accurately assess whether the ARCD was perforated using CT images. A higher percentage of grade II cases were identified in the surgical group, despite a preoperative CT assessment of grade I. CT imaging appears insufficient for accurately assessing Hinchey's classification of ARCD. The Chinese literature provides many cases of colonic diverticulum perforation, most commonly occurring in the sigmoid colon $[5,6]$. This may be due to the protection offered by the greater omentum. ARCD may have been wrapped with the greater omentum before suppuration and perforation. The sigmoid colon is not easily wrapped by the omentum, and patients with sigmoid diverticulitis are generally older. 
ARCD is often accompanied by increases in inflammation indicators. Some patients with mild symptoms may have a normal inflammation index. The sensitivity of CRP is relatively high, while the sensitivity of WBC is relatively low. If the inflammation index is not high, the patient can recover quickly without special treatment. MÄKELÄ et al. published a study showing that CRP > $150 \mathrm{mg} / \mathrm{L}$ is an independent risk factor for colonic diverticulitis [7]. In our study, the proportion of patients with CRP $>150 \mathrm{mg} / \mathrm{L}$ was relatively low. Simultaneously, there was no significant increase in CRP at early stages, and this value did not play an important reference role in treatment decisions. However, the CRP index is an important reference value for predicting treatment outcome. CT imaging can quickly and effectively evaluate the severity of diverticulitis, and patients with limited inflammation usually recover better.

No significant difference in patient outcome was observed between our three treatment groups. However, the conservative, non-surgical treatment was much less expensive. Therefore, we recommend a more conservative treatment approach, which is also consistent with the results of other studies [1]. At present, the most commonly used surgical method is colectomy, but that surgery produces significant trauma [8-10]. Currently, there are no detailed guidelines for the treatment of ARCD. In China, colonic diverticulitis often occurs in the cecum and ascending colon. Right hemicolectomy may be required $[11,12]$. Most of the cases in our hospital underwent resection and repair of colonic diverticulum or abdominal drainage. Post surgery, there was no intestinal leakage, and the postoperative recurrence rate was low. Colectomy is not recommended for uncomplicated diverticulitis. Is it feasible to repair acute complicated diverticulitis? We lack enough patients with complicated diverticulitis to study the feasibility of diverticulectomy and repair of sigmoid diverticulitis. In our hospital, only one patient with uncomplicated sigmoid diverticulitis underwent diverticulum resection, and did not experience intestinal leakage following surgery.

In our research, we found that the rate of recurrence of ARCD was low with relapse usually occurring relapsed within the first year. Colonoscopy is not recommended during hospitalization because it may aggravate the condition. Colonoscopy is routinely recommended 2-3 months after discharge; however some patients did not undergo colonoscopy. In China, a high proportion of younger people with ARCD refused colonoscopy.

\section{Conclusions}

In summary, ARCD is more common in young and middle-aged men. In China, ARCD is more common than ALCD, and acute right-sided complicated diverticulitis is rare. The clinical symptoms of ARCD are very similar to acute appendicitis, and can be easily misdiagnosed. While drainage of abdominal abscesses or diverticulum resection and repair are effective for acute uncomplicated diverticulitis, we recommend a more conservative, non-surgical treatment for acute uncomplicated diverticulitis. While the inflammation index was useful for predicting treatment outcome, abdominal CT proved more useful for diagnosing colonic diverticulitis."

\section{Abbreviations \\ CT: Computed tomography; CRP: C-reactive protein; WBC: White blood cell; ALCD: Acute left-sided colonic diverticulitis; ARCD: Acute right-sided colonic diverticulitis; WSES: World Society of Emergency Surgery}

\section{Acknowledgements}

Thanks to all general surgeons for their help. Thanks to all patients who cooperated with the follow-up survey.

\section{Authors' contributions}

CZ: mainly collects data and writes articles. WD: analyze data and assist with writing articles. ZB: provides research content and analyzes the latest literature reports. JY: assists in collecting data and excluding inappropriate cases. All authors read and approved the final manuscript.

Funding

Non-funding.

Availability of data and materials

All data generated or analysed during this study are included in this published article [and its supplementary information files].

Ethics approval and consent to participate

The study was approved by the Ethics Committee of Zhejiang Tongde Hospital.

Consent for publication

Not applicable.

\section{Competing interests}

The authors declare that they have no competing interests.

\section{Author details}

${ }^{1}$ Department of General Surgery, Tongde Hospital of Zhejiang Province, Hangzhou 310000, China. 'Department of Anesthesiology, Zhejiang Hospital, No.1229, Gudun Road, Xihu District, Hangzhou city 310030, Zhejiang province, China.

Received: 26 May 2020 Accepted: 7 September 2020

Published online: 14 September 2020

\section{References}

1. Neil S, Walter S, Ikuo H, et al. American Gastroenterological Association Institute guideline on the Management of Acute Diverticulitis [J]. Gastroenterol. 2015;149(7):1944-49.

2. Massimo S, Dieter GW, Yoram K, et al. 2020 update of the WSES guidelines for the management of acute colonic diverticulitis in the emergency setting [J]. J Emerg Surg. 2020;15(1):385-9.

3. Xin-Ying Z, Wen-Juan Z, Juan D, et al. Clinical characteristics of 132 patients with acute colonic diverticulitis [J]. China J Endoscopy. 2019;25(7):30-33.

4. Fang Y, Kui J, Chao S, et al. Prevalence and growth trend of colonic diverticulosis in northern China [J]. China J Endoscopy. 2019;25(10):37-41.

5. Chuan-Dong K, Hong-Xing Z, Xin-Guang C. Surgical treatment of acute perforation induced by colonic diverticulitis: clinical analysis of 4 cases [J]. J. Abdom Surg. 2002:15(3):158-9.

6. Sun-Long Y, Qing Y, Zhi-Guo W, et al. Diagnosis and treatment experience of sigmoid diverticulum perforation [J]. Zhejiang J Traumat Surg. 2015; 20(02):242-3. 
7. Mäkelä JT, Klintrup K, Takala H, et al. The role of C-reactive protein in prediction of the severity of acute diverticulitis in an emergency unit [J]. Scand J Gastroenterol. 2015;50(5):536-41.

8. Hong MK, Tomlin AM, Hayes IP, et al. Operative intervention rates for acute diverticulitis: a multicentre state-wide study [J]. ANZ J Surg. 2015;85(10):7348.

9. Daniël PVL, Sandra V, Gijsbert DM, et al. Hartmann's procedure versus sigmoidectomy with primary anastomosis for perforated diverticulitis with purulent or faecal peritonitis (LADIES): a multicentre, parallel-group, randomised, open-label, superiority trial [J]. The lancet. Gastroenterol Hepatol. 2019;4(8):599-610.

10. Jae ML, Jun BPC, Majed EH, et al. Hartmann's procedure vs primary anastomosis with diverting loop ileostomy for acute diverticulitis: Nationwide analysis of 2,729 emergency surgery patients [J]. J Am Coll Surg. 2019;229(1):48-55

11. Hao C, Tian-Sheng C, Jian W, et al. Diagnosis and treatment of acute abdominal disease caused by colonic diverticulum [J]. Chin Arch GeneraSurg (Electronic Edition). 2019;13(05):381-4.

12. Tse-Cheng C, Yi-Hong C, Chui-Mei T, et al. Right-sided colonic diverticulitis: clinical features, sonographic appearances and management [J]. J Med Ultrasound. 2017;25(1):33-9.

\section{Publisher's Note}

Springer Nature remains neutral with regard to jurisdictional claims in published maps and institutional affiliations.

Ready to submit your research? Choose BMC and benefit from:

- fast, convenient online submission

- thorough peer review by experienced researchers in your field

- rapid publication on acceptance

- support for research data, including large and complex data types

- gold Open Access which fosters wider collaboration and increased citations

- maximum visibility for your research: over $100 \mathrm{M}$ website views per year

At $\mathrm{BMC}$, research is always in progress.

Learn more biomedcentral.com/submissions 